\title{
Anabases
}

ANABASES Traditions et réceptions de l'Antiquité

$6 \mid 2007$

Varia

\section{Caylus e le pitture ercolanesi}

\section{Enrica Pagano}

\section{OpenEdition}

\section{Journals}

Edizione digitale

URL: http://journals.openedition.org/anabases/3348

DOI: 10.4000/anabases.3348

ISSN: 2256-9421

\section{Editore}

E.R.A.S.M.E.

\section{Edizione cartacea}

Data di pubblicazione: 1 ottobre 2007

Paginazione: 113-134

ISSN: 1774-4296

\section{Notizia bibliografica digitale}

Enrica Pagano, «Caylus e le pitture ercolanesi », Anabases [Online], 6 | 2007, Messo online il 01 janvier 2012, consultato il 20 octobre 2019. URL : http://journals.openedition.org/anabases/3348; DOI :

10.4000/anabases.3348 
Anabases 6 (2007), p. 113-134.

\section{Caylus e le pitture ercolanesi}

ENRica Pagano

\section{Gli scavi di Ercolano : un inaccessibile " jardin des hespérides "}

La PASSIONe PER LE ANTICHITÀ del conte di Caylus risale all'epoca del Voyage d'Italie (1714-1715). Roma, come pure l'Italia meridionale, ebbero un ruolo determinante nella sua formazione ${ }^{1}$. L'anno seguente intraprese un nuovo viaggio, che lo portò nella Troade e nell'Asia Minore occidentale, spinto da un più spiccato interesse archeologico ${ }^{2}$. Il valore del racconto del viaggio in Italia, depositato dal conte nelle mani dell'amico Mariette e non destinato alla pubblicazione, risiede principalmente nella possibilità di confrontare le annotazioni riportate da Caylus con altri episodi della sua vita e della sua attività e con il contesto culturale dell'epoca ${ }^{3}$.

Così scrive Caylus di Ercolano : "Dans un petit village au bord de la mer, à une lieue de Naples, et presqu'au pied du Vésuve, le prince Emmanuel d'Elbeuf qui s'est marié à Naples, a fait bâtir une petite maison assez jolie. La vue et la situation en font

1 Lo confermano queste parole scritte a Paciaudi : "J'éprouve tous les jours que le germe qu'on acquiert à Rome et dans quelques autres villes d'Italie jette des racines si profondes qu'elles survivent pour ainsi dire à celui qui le porte ", in CAYLuS, Correspondance inédite $d u$ comte de Caylus avec le p. Paciaudi, théatin (1757-1765) suivie de celles de l'abbé Barthélemy et de P. Mariette avec le même, a cura di Charles Nisard, I, Paris, 1877, p. 142.

2 Cf. CAYlus, "Voyage de Constantinople", a cura di P.-E. Schazmann, Gazette des BeauxArts 897 (1938), p. 272-292 ; 899 (1938), p. 111-126 ; 902 (1938), p. 309-322.

3 Il conte, di certo ben accolto dalla comunità francese di Napoli, nel 1715 visita i primi saggi di scavo di Ercolano, intrapresi dal principe d'Elboeuf, e in quell'occasione probabilmente lo incontra. 
l'agrément. Pas loin de cette maison le dit prince d'Elbeuf s'étant mis en teste de faire creuser, l'a fait faire dans le puit d'un paysan au fond du quel il a trouvé des marbres, des murs, un degré, des statues très belles qu'il a envoyées au prince Eugène. Il a très peu creusé, cependant il a beaucoup tiré. Les antiquaires ont baptisé cela les ruines de l'ancienne ville d'Herculanum qui, devant la venue de Notre Seigneur, fut engloutie par un tremblement de terre auquel se joignit l'embrasement du Vésuve. Effectivement j'ai vu plusieurs des pierres que l'on a tirées qui sont brûlées. Les échaufauds estant défaits, j'avois eu l'idée de faire la dépense pour les remettre, mais je me contentois de l'envie sur ce qu'il me fut dit du peu de satisfaction que j'en retirerois ${ }^{4}$ ».

Caylus dunque intuisce immediatamente l'importanza della città sepolta dal Vesuvio, progettando addirittura di riaprire i lavori a proprie spese. Atteggiamento sorprendente se si considera la sua giovane età e il fatto che questi primi scavi ercolanesi sono completamente ignorati da altri viaggiatori stranieri come Berkeley (1717), Delamonce (1719) e Montesquieu (1720).

L'interesse per Ercolano si accende quando, intrapresa una regolare campagna di scavi nel 1738, per iniziativa di Carlo di Borbone, che gli esiti della guerra di successione polacca avevano portato al trono delle due Sicilie, e del suo ministro Tanucci, si cominciano a diffondere notizie sui sorprendenti risultati di essi. Tra i primi a visitarli, nel novembre 1739, è il presidente de Brosses ${ }^{5}$, che scende attraverso il pozzo del principe d'Elboeuf nella zona della città sepolta, in cui si trovano i resti del teatro e dell'attigua Basilica, considerando sin d'allora le persone che mostravano le antichità gelose $\mathrm{e}$ poco disponibili.

I risultati degli scavi furono, com'è noto, sottoposti a segreto dal ministro Tanucci, per volontà del sovrano, impedendo persino che i pochi, cui veniva consentito vederli, ne potessero trarre schizzi o disegni ; la sottrazione di reperti fu oggetto di sanzioni severissime, come poterono sperimentare nel 1740 alcuni rei confessi, sotto tortura, di aver rubato pochi oggetti di insignificante valore. Persino al grande Winckelmann la possibilità di visitare a suo agio i reperti ercolanesi custoditi nel museo di Portici fu consentita non senza difficoltà ; Goethe $\mathrm{e}$ Tischbein non riuscirono a ottenere l'autorizzazione a disegnare sui luoghi ; al teatino Paciaudi, insigne studioso, corrispondente e fidato collaboratore di Caylus, fu da Tanucci interdetto l'accesso al museo, perché accusato dagli accademici ercolanesi di prendere nota di tutto, con il pericolo di diffondere notizie sulle antichità ercolanesi prima ancora che fossero pubblicate ${ }^{6}$.

4 CAYLus, Voyage d'Italie 1714-1715, première édition du code autographe annotée et précédée d'un essai sur le comte de Caylus par Amilda-A. Pons, Paris, 1914, p. 255. De Brosses ne parla nella Lettre au Président Boubier, XXXIII del 28 novembre 1739, in Lettres familières, edizione a cura di Y. Berard, Paris, 1931, p. 464-476.

6 Cf. lettera LXVII di Paciaudi a Caylus del 3 novembre 1761, in P. M. PACIAUDI, Lettres de Paciaudi au comte de Caylus, a cura di A. Sérieys, Paris, 1802, p. 270. 
L'imposizione del segreto riuscì, come a molti, affatto incomprensibile a Caylus, uomo del nuovo secolo dei lumi, sempre prodigo a rendere gli altri partecipi dei risultati dei propri studi e a consentire a tutti la fruizione delle proprie raccolte di oggetti antichi, studiati e poi donati alle collezioni reali ${ }^{7}$. "Le vilain Tanucci 8 " è oggetto di ripetuti attacchi da parte del conte nella corrispondenza con Paciaudi e l'astio si estende ai napoletani, che "sont des barbares et ce sera toujours mon refrain 9 " ; considerate le difficoltà di avere " des fruits de ce jardin des Hespérides 10 ", egli si contenterebbe anche di piccoli oggetti di uso comune, di solito trascurati ; per l'impossibilità di ottenerne, nonostante i buoni uffici interposti da Barthélemy e Paciaudi, arriva addirittura a progettarne il furto ${ }^{11}$. Quanto alle pitture, riesce a ottenere, per via traversa, un frammento di pittura parietale e quello portatogli da Soufflot, entrambi inclusi nella sezione delle antichità greche del I tomo del Recueil, e piccoli frammenti di muri ercolanensi che, sempre nel medesimo tomo, riferisce di avere esaminati. Per il resto, si tratta di disegni, frettolosamente eseguiti a memoria da visitatori non appena lasciato il museo e spesso male incisi.

Ben poca cosa per chi, come Caylus, è aduso all'osservazione " scientifica " dei resti, attraverso " la voie de comparaison, qui est pour l'Antiquaire ce que les observations et les experiences sont pour le Phisicien 12 ". Seguiranno solo vaghe promesse, come quelle, puntualmente non mantenute, dell'abate Galiani, segretario dell'ambasciata napoletana a Parigi ${ }^{13}$, o indicazioni di vie considerate non percorribili, come quella suggerita da Paciaudi, di rivolgersi al duca di Choiseul per premere sul re ${ }^{14}$, o quella di Taitbout, console di Francia a Napoli, di accattivarsi le simpatie dell'ingegnere Bardet de Villeneuve, di recente nominato primo direttore dei lavori di Ercolano ${ }^{15}$.

7 Rinviamo alla nostra comunicazione "Caylus Mécène du Roi. Collectionner les Antiquités aux XVIII siècle”, Cronache Ercolanesi 34 (2004), p. 209-211, in margine all'omonima esposizione tenuta dal 17 dicembre 2002 al 17 marzo 2003, presso il Musée des monnaies, médailles et antiques de la Bibliothèque Nationale de France, site Richelieu, a Parigi.

8 Lettera LXIII del 20 giugno 1763 in CAYlus, Correspondance, I, p. 320.

$9 \quad$ Lettera LIII del 12 ottobre 1761 in CAYLUS, Correspondance, I, p. 261.

10 Lettera XXVI del 25 dicembre 1759 in CAYLUS, Correspondance, I, p. 116.

11 Cf. lettere XXIII del 13 dicembre 1759, XXV del 17 dicembre 1759 e XXVI del 25 dicembre 1759, in CAYLUS, Correspondance, I, p. 101-102, 112, 114 e 116.

12 CaYlus, Recueil d'antiquités égyptiennes, étrusques, grecques et romaines, I, Paris, 1752, p. 11.

13 Lettere CXXXVIII del 23 giugno 1765, p. 141 ; CXLI del 15 luglio 1765, p. 147-148; CXLII del 22 luglio 1765, p. 153 ; CXLV del 11 agosto 1765, p. 161, in CAYLUS, Correspondance, II.

14 Lettera XXV del 9 gennaio 1760, in P. M. PACIAUDI, Lettres, p. 108.

15 Cf. lettera VI di Taitbout del 10 agosto 1751, in Lettres inédites de Henri IV et de plusieurs personnages célèbres, tels que Fléchier, La Rochefoucauld, Voltaire, le comte de Caylus, Anquetil-Duperron, etc. Ouvrage dans lequel se trouvent éclaircis plusieurs points d'histoire très curieux, a cura di A. Sérieys, Paris, 1802, inviata al conte da Napoli. 
Tuttavia anche Caylus finisce nel mirino delle autorità napoletane. Paciaudi, nella lettera LII del 1 agosto $1761^{16}$, gli comunica di aver appreso dal cavaliere Basquiat, ministro di Francia, che il governo napoletano è " très-faché " perché il conte ha affermato nella sua opera di essere in possesso di oggetti provenienti da Ercolano ${ }^{17}$ e gli suggerisce di inserire nel prossimo IV tomo del Recueil una dichiarazione di apprezzamento delle misure adottate da Tanucci per la custodia del museo « et tout sera accomodé ». Ma l'invito, reiterato nella successiva lettera LXIV del 12 settembre 1761 18, rimarrà inascoltato. Anzi Caylus, nell'inviare a Paciaudi la prefazione al IV tomo, lo invita ad aggiungere « quelques choses qui puissent le (scil. Tanucci) piquer et le fâcher, mais toujours sur le ton doux et poli que j'ai pris 19 ".

Le poche notizie che filtrano sui reperti ercolanensi e le scarse conoscenze che se ne hanno alimentano poi, indirettamente, la fiorente industria dei falsi e il singolare rimedio per qualche tempo adottato dalle autorità napoletane di distruggere gli intonaci di poco interesse perché non possano essere utilizzati come supporto dei falsi. In essi primeggia il veneziano Giuseppe Guerra, autore di falsi frammenti di pitture su intonaco, che asseriva essere in parte provenienti da Ercolano o Pompei, riuscendo a trarre in inganno, oltre ad altolocati acquirenti, persino Mengs, a sua volta autore di un Giove e Ganimede a imitazione degli antichi, che lo stesso Winckelmann ritenne per autentico ; e anche Caylus, a quanto si deduce dalla sua lettera a Paciaudi XLIII del 22 settembre $1760^{20}$, fece disegnare e incidere due quadri di Guerra ritenendoli ercolanesi ; fatto questo rilevato da Winckelmann nelle sua lettera al conte di Brühl del 1762 e negato da Caylus in una nota alla traduzione in francese di tale lettera da lui stesso fatta eseguire due anni dopo ${ }^{21}$. E il sospetto dilaga e finisce con l'investire anche il padre Contucci, guardia del Museo del Collegio romano, possessore di diversi quadri " ercolanesi " del Guerra : Paciaudi dichiara al conte di ignorare se il gesuita sia in buona o cattiva fede 22 ; Caylus, nel IV tomo del Recueil, conclude « je ne le crois pas capables d'un procédé si odieux 23 "; tutto sommato al gesuita va bene questa poco convinta assoluzione, tenuto conto dell'ostilità, del conte e del teatino, all'ordine cui egli appartiene.

P. M. Paciaudi, Lettres, p. 254.

Probabilmente si tratta dell'affermazione contenuta nel III tomo del Recueil (1759, p. 140) di aver eluso la sorveglianza dei dragoni di Tanucci.

P. M. PACIAUdi, Lettres, p. 260.

Lettera LXIII del 20 giugno 1763 in CAYLus, Correspondance, I, p. 320.

CaYlus, Correspondance, I, p. 208.

De Vos ha ritenuto falso, probabilmente ad opera di Guerra, il dipinto egittizzante riportato alla tavola II del volume VII del Recueil, di cui Caylus non indica altri dati, se non una vaga provenienza da Marsiglia (CAYLUS, Recueil, II) ; cf. M. DE Vos, "La ricezione della pittura antica", in S. Settis (a cura di), Memoria dell'antico nell'arte italiana, II, I generi e i temi ritrovati, Torino, 1985, p. 354-377.

Lettera XLII del 2 settembre 1760, in P. M. PACIAUdi, Lettres, p. 171.

CAYLUS, Recueil, IV, 1761, p. 220. 
Il quadro sinora tracciato non è certamente propizio per gli studi su Ercolano ; esso non consente un approccio serio e approfondito ai risultati degli scavi e, meno che mai, alle pitture parietali, delle quali, con la sola diffusione dei disegni, finisce con lo sfuggire l'aspetto fondamentale del colore.

\section{Le pitture ercolanesi : fonti e limiti delle conoscenze di Caylus}

Le principali fonti da cui Caylus attinge notizie sugli scavi ercolanesi sono i pensionnaires dell'Accademia di Francia a Roma, Cochin il giovane, Soufflot, Barthélemy e Paciaudi 24.

Quanto ai primi, Petitot, Vien, Bellicard e Tiersonnier avevano visitato Ercolano e Petitot si era liberamente ispirato al teatro ercolanese per la seconda macchina di fuochi d'artificio della festa della Chinea del 29 giugno 1749. Caylus menziona i pensionnaires, che la curiosità ha condotto a Portici, nel I tomo del Recueil, a proposito di quel primo frammento di pittura parietale pubblicato, da essi giudicato pari, per disegno e colore, a quelli visti sul posto. D'altro canto, sono noti i buoni rapporti tra Caylus e i giovani ospiti dell'Académie, Petitot in particolare, che in più occasioni collaborò con il conte e a lui dové la nomina ad architetto del ducato parmense.

La fonte più ricca e appropriata, per la competenza dell'autore, è quella di Cochin, che al seguito di M. de Vandières, poi marchese di Marigny, insieme all'architetto Soufflot e al critico Le Blanc, eseguì il Grand Tour d'Italie (1749-1751) e nel 1750, recatosi a Napoli, ebbe occasione di vedere le pitture parietali distaccate da Ercolano e esposte nella Reggia di Portici. È a Cochin che deve attribuirsi la paternità di una lettera anonima, datata 20 gennaio 1751 (Bruxelles), poi pubblicata sul Mercure de France nel settembre dello stesso anno, di cui sarebbe destinatario proprio Caylus. L'autore scrive di aver fatto stampare 12 esemplari della lettera, anche perché ne ha promesso una copia ad un imprecisato amico tedesco. Potrebbe trattarsi di Winckelmann, il quale richiamò il giudizio negativo sulle pitture staccate dal teatro di Ercolano di un artista che aveva avuto modo di vederle direttamente (forse proprio Cochin), rilevando dal suo canto come le grandi figure fossero in parte mediocri e in parte mal disegnate e le teste mancanti di espressione ${ }^{25}$. Ad avvalorare l'ipotesi che l'amico tedesco fosse

24 Sembra che Caylus non avesse letto la relazione di de Brosses, né il testo di Venuti dal quale il Presidente aveva attinto numerose notizie ; in tal senso cf. L. NorCI CaGianO, "Caylus en Campanie", Journal des Savants, 2000, p. 124-140.

Cf. J. J. WinCKELMANN, Gedanken über die Nachahmung der griechischen Werke in der Malerey und Bildhauerkunst, Dreisden, 1755. In verità, dopo averne avuto diretta visione, il tedesco rivide i propri giudizi ; cf. il cap. Ercolano, in C. LENZA, "La cultura architettonica e le antichità. Scavi, rilievi, restauri, editoria antiquaria e dibattito teorico", estratto anticipato da A. Gambardella (a cura di), L'architettura dei Borbone di Napoli e Sicilia, Napoli, in corso di stampa. 
Winckelmann depone la coincidenza dei soggetti ercolanesi descritti da entrambi. I disegni sono stati eseguiti a memoria, dopo la visione diretta delle pitture antiche conservate nel palazzo reale. Durante questa visita, l'autore ha riscontrato prove inconfutabili della fondatezza di alcune tesi del destinatario della lettera sulla pittura degli antichi e delle congetture di questi su Ercolano, riportate in una memoria letta all'Accademia delle Iscrizioni l'anno precedente. L'autore deride poi l'abuso di superlativi da parte degli Italiani nel descrivere le proprie antichità e in particolare ridicolizza Venuti, quando afferma che gli antichi ben conoscevano la prospettiva, superavano Raffaello nel disegno e facevano vergognare Tiziano nel colore. Riprende la riflessione di un amico, non precisato, del destinatario, secondo cui Ercolano è città di provincia, nella quale certamente non hanno lavorato i grandi artisti del tempo ; affermazione peraltro già fatta da Caylus nella memoria De la perspective des Anciens del 1749 e ripetuta poi nel Recueil. Riconosce comunque che le sue pitture sono di grande interesse. Mette in guardia sull'affidabilità delle prossime pubblicazioni su Ercolano fatte a Napoli, preoccupandosi di come le pitture saranno riportate, per effetto della correzione degli errori di prospettiva contenuti nei dipinti. Descrive in dettaglio alcune pitture, scelte in base alla maggiore complessità della raffigurazione. I soggetti non sono certi e preferisce riportare le attribuzioni correnti nell'ambiente napoletano ${ }^{26}$. Circa la prospettiva, all'autore non sembra che i pittori di Ercolano ne fossero istruiti, come provano, tra gli altri, i dipinti raffiguranti elementi architettonici. I pittori non avrebbero rappresentato quel che vedevano, ma espresso queste fabbriche con cattivo gusto e in maniera stravagante, da lui stesso definita come gotica. Quanto al disegno, l'autore osserva che la maniera di disegnare secca è principalmente dovuta al cattivo uso dello studio delle statue, che, concepite per stare da sole, non si adattano alla composizione. Per quanto concerne i colori, le mezze tinte sono olivastre, giallastre e rosseggianti, le ombre di un rosso mescolato con il nero, non c'è poi chiaroscuro, e quindi né armonia, né consonanza. Ogni figura ha la sua luce e la sua ombra e appare isolata. Nonostante tutto, il tocco appare leggero, il pennello maneggiato con libertà, pressappoco alla stessa maniera con cui vengono dipinte le decorazioni teatrali. Le composizioni più piccole presentano una più riuscita disposizione delle figure e un miglior uso del colore. Frutti, fiori e vasi sono ben imitati, ma inferiori nei colori. Conclude dicendo che questi pittori furono certamente deboli se confrontati a quelli attivi nelle grandi città, pur dovendosi nei loro dipinti riconoscere i segni delle varie componenti dell'Arte. Si pone infine l'interrogativo di come possa conciliarsi ad Ercolano la qualità dell'architettura e della scultura con la mediocrità della pittura e ritiene che, pur poten-

26 Le cinque tavole prese in esame raffigurerebbero : I) Minotauro morto ai piedi di Teseo ; II) donna seduta e Ercole ; III) il Centauro Chirone che insegna la lira al giovane Achille ; IV) giudizio di Appio ; V) giudizio di Paride. 
dosi dare varie risposte, altri siano maggiormente qualificati a farlo, rivendicando però a sé di aver riportato con verità e onestà le proprie osservazioni 27 .

Dal contenuto appare chiaro il riferimento alla memoria di Caylus sulla prospettiva degli antichi del 1749 e l'identità del destinatario della lettera, che sarebbe dunque Caylus stesso. La tesi sostenuta da alcuni, secondo cui il conte sarebbe invece autore della lettera, è smentita da molteplici elementi. L'autore afferma chiaramente di aver visitato i dipinti ercolanesi nella reggia di Portici, il che già di per sé porta ad escludere la paternità di Caylus, considerato che il suo viaggio in Italia è avvenuto molti anni prima (1714-1715), quando i dipinti stessi non erano stati ancora portati alla luce. D'altro canto, lo stesso Caylus riconosce, nel tomo III del Recueil, di data posteriore rispetto alla lettera, di non aver mai preso diretta visione dei dipinti ercolanesi, ma di averli conosciuti soltanto attraverso incisioni ${ }^{28}$. Ancora, alcune affermazioni contenute nella lettera, specie in ordine agli errori di prospettiva addebitati alle pitture ercolanesi, non si conciliano con le considerazioni svolte in proposito da Caylus nel De la perspective des Anciens. Persuasiva appare, invece, l'attribuzione della lettera a Cochin, il quale ritornerà sull'argomento delle pitture ercolanesi nelle Observations sur les antiquités d'Herculanum, scritte insieme a Bellicard e pubblicate la prima volta in inglese nel 1753, comparse in francese l'anno seguente e riedite in due riprese (1755 e 1757). Le analogie tra la lettera in questione e questa opera sono tali da non poter mettere in dubbio l'attribuzione a Cochin ${ }^{29}$. Le tavole descritte in dettaglio sono le stesse. E analoghi sono i giudizi che ne vengono formulati : il maldestro utilizzo delle statue come modello, la maniera di dipingere simile alla decorazione teatrale, i difetti nell'utilizzo dei colori, la mancanza di proporzioni, il cattivo gusto dei dipinti raffiguranti architetture o rovine. L'attribuzione a Cochin spiega anche perché la lettera porti l'indicazione Bruxelles e non sia firmata dall'autore. Nel gennaio del 1751, Cochin si trovava ancora in Italia, impegnato nel viaggio a seguito di M. de Vandières, terminato nell'agosto dello stesso anno, e non voleva urtare la sensibilità dei vari ospiti italiani. Di qui l'indicazione fittizia di Bruxelles, per fuorviare eventuali sospetti sull'autore.

Altre notizie certamente sono fornite a Caylus da Soufflot, che al Grand Tour partecipò dal dicembre 1749 all'autunno 1750, abbandonandolo per ragioni di salute, mentre per i compagni, con l'aggiunta di Bellicard, il viaggio si protrasse come previsto

27 Le lettera è pubblicata nell'appendice a J.-J. BARTHÉLEMY, Voyage en Italie de M. L'Abbé Barthélemy, de l'Académie Française, de celle des Inscriptions et des Belles-Lettres, et auteur du Voyage d'Anacharsis, imprimé sur ses lettres originales écrites au Comte de Caylus, a cura di A. Sérieys, Paris, 1802, p. 270-286, con la seguente premessa del curatore : "La lettre suivante est addressée au comte de Caylus ; elle est écrite et corrigée de sa main. Les observations critiques qu'elle contient, au sujet des peintures d'Herculanum, m’ont parée mériter une place à côté des jugemens de l'abbé Barthélemy et d'autres savans. » amateurs, critiques, de Poussin à Diderot, Paris, 1909 ; ed. consultata : Genève, 1989. 
fino al settembre 1751. I rapporti di Soufflot con Caylus furono assai stretti già in quegli anni, durante i quali l'architetto lionese raccolse notizie e disegni ; diverse le prove che ne emergono dagli scritti del conte. A quell'epoca risalgono i disegni di una " mardelle de puits " in marmo rinvenuta ad Ercolano che Soufflot fornì a Caylus e che questi pubblicò nel IV tomo della sua raccolta di antichità ${ }^{30}$, riferendo che l'amico ne aveva viste altre simili, tutte di buona fattura. La lettera LIII a Paciaudi del 12 ottobre $1761^{31}$, nella quale, in relazione allo stesso reperto ercolanese, si legge " mesuré et dessiné dans les fouilles par un architecte des mes amis qui était à Naples avec M. de Marigny ", chiarisce ulteriormente il loro rapporto. E fu Soufflot a fornire a Caylus il già ricordato frammento di pittura riportato nel I tomo del Recueil.

Altre notizie su Ercolano furono fornite nell'anno 1756 da Barthélemy, che si trovava allora in Italia, il cui Voyage en Italie è in sostanza la pubblicazione della sua corrispondenza con il conte di Caylus ${ }^{32}$. In particolare la lettera XIII del 2 febbraio 1756, dove, a seguito delle numerose visite effettuate nel cabinet di Portici, descrive al conte $\mathrm{i}$ dipinti che più hanno suscitato la sua attenzione. Anche Barthélemy, nonostante i buoni rapporti con le autorità e gli eruditi napoletani, incontra difficoltà a documentarsi sui dipinti e scrive in proposito " que dans ce sanctuaire respectable, il n'est permis que de rassasier sa vue, et qu'on revient à Naples, les tablettes vides et la mémoire pleine 33 ». Barthélemy avverte la mediocrità di molte pitture sotto il profilo artistico : "Je sais bien qu'il y a, dans les cabinets de Portici, bien de morceaux médiocres aux yeux d'un artiste ; mais je pense, qu'aux yeux d'un homme de lettres, il doivent être précieux 34 ». Tuttavia è l'aspetto letterario quello che, a differenza di Cochin, lo interessa : «je ne vous dirai rien des tableaux considérés relativement à l'art : je les ai examinés par rapport à la littérature 35 ». E mentre Cochin accentra la sua indagine sui generi delle pitture in ordine decrescente di importanza, Barthélemy si sofferma piuttosto sull'insieme di esse, con i relativi richiami storici e letterari. Si intrattiene in particolare sui dipinti relativi agli usi religiosi degli egiziani, preziosa testimonianza, a suo giudizio, della connessione cultuale tra Egitto e Grecia, già incisi nel Recueil di Cochin e Bellicard, seppure con qualche inesattezza, probabilmente dovuta a quella fretta imposta dai custodi, di cui lo stesso Cochin si lamenta nella Lettre; sulle nove tavole rappresentanti Apollo e sulle otto dedicate alle Muse ; sui « quatre ou cinq petits dessins sur marbre, qui, au sentiment de plusieurs artistes, sont faits avec plus de soin que la plupart des peintures d'Herculanum 36 ".

CAYlus, Recueil, IV, p. 173.

31 CaYlus, Correspondance, I, p. 263.

32 Cf. J.-J. BARTHÉLEMY, Voyage, p. 77-89.

33 J.-J. BARTHÉLEMY, Voyage, p. 77.

34 J.J. BARTHÉLEMY, Voyage, p. 78.

35 J.J. BARTHÉLEMY, Voyage, p. 79.

36 J.-J. BARTHÉLEMY, Voyage, p. 81.
} 
Come sempre preziosa la collaborazione di Paciaudi, che si trattenne a Napoli dal luglio 1761, ospite del principe di Francavilla, gran maestro della Casa del re di Napoli, per cinque mesi. Egli visitò per otto giorni Ercolano e il museo di Portici e, nella lettera LXIV del 12 settembre $1761^{37}$, dichiara : «j'ose dire que j'ai plus appris dans ces huit jours, en parcourant des yeux ce muséum, qu'en étudiant dix ans, et que celui qui n’a point vu les monumens d'Herculanum manque d'une infinité de connoissances. Et pourtant il est défendu de parler d'Herculanum. Le roi catholique veut qu'on admire et qu'on se taise, qu'on aille à Herculanum comme à la Mecque. Quelle loi barbare! ». Gli comunicherà poi, con la già citata lettera del 3 novembre successivo ${ }^{38}$, che Tanucci gli ha interdetto l'ingresso al museo, raccomandando al conte di non parlarne con Galiani, «l'espion de Tanucci ».

\section{Il giudizio di Caylus}

\section{1. Ercolano e Roma : i dipinti di una città di provincia}

I quadri di grandi pittori greci del IV e III secolo, portati a Roma come bottino di guerra dopo la presa di Siracusa (212 a.C.), la caduta di Corinto (146 a.C.) e la conquista della Macedonia (146 a.C.) e sistemati in strutture pubbliche, erano andati nel tempo scomparendo specie per la deperibilità del supporto ligneo. Nel De la perspective des Anciens Caylus lamenta che la pittura antica di maggiore qualità non sia arrivata fino a noi, osservando come, già ai tempi di Augusto, i dipinti di Zeuxis, Apelle, Protogene e altri grandi pittori fossero fortemente danneggiati, anche per il degrado delle tavole lignee, che, a quanto riferito dagli storici, erano le uniche usate. Di questi dipinti non era rimasta alcuna traccia, salvo qualche copia o imitazione in pitture murali. Plinio ricorda in proposito che in antico non v'era gloria che per gli artisti che avevano dipinto quadri (sed nulla gloria artificum est nisi qui tabulas pinxere, eo venerabilior antiquitatis prudentia apparet. Non enim parietes excolebant dominis tantum, nec domos uno in loco mansuras, quae ex incendiiis rapi non possent ${ }^{39}$ ); la grande arte, già espressa nei quadri, era al suo tempo prossima a morire 40 ; una volta bastavano quattro semplici colori per creare opere immortali, mentre al presente si ricercavano materie coloranti preziose per dipingere le pareti, più per ostentarne il valore che non per gusto artistico ${ }^{41}$. Lo stesso Caylus afferma, nel II tomo del Recueil, che « chez les Grecs

P. M. PACIAUDI, Lettres, p. 260.

P. M. PaCiaudi, Lettres, p. 270.

Plinio, Naturalis Historia XXXV, 118.

Plinio, Naturalis Historia XXXV, 28.

41 Plinio, Naturalis Historia XXXV, 50. 
la peinture entroit dans les décorations des temples, des portiques, des tombeaux ; mais il me paroit prouvé qu'il l'ont moins cultivée que les autres arts. Pausanias ne fait mention que de quinze peintres, tandis qu'il distingue cent soixante neuf sculpteurs 42 ». Non rimane dunque che la pittura parietale, di carattere prevalentemente decorativo, considerata espressione minore dell'arte pittorica rispetto a quella delle tabulae dipinte, della quale nel Settecento si conoscevano nella capitale pochi e mal conservati esempi : le tombe (piramide Cestia, vigna Codini, tomba dei Nasoni), le tracce delle grottesche della Domus Aurea neroniana, i frammenti nelle collezioni Albani, Barberini, Massimo e soprattutto le celebri Nozze Aldobrandini, considerate l'espressione migliore della pittura parietale antica. Ed è proprio a queste, scoperte all'Esquilino nel 1606, ammirate da Rubens e copiate da Van Dyck, Pietro da Cortona e Poussin, che Caylus, nel De la perspective des Anciens, riserva particolare attenzione, esaltandone la semplicità e la nobiltà della composizione, i toni dei colori tipici dell'affresco, il tocco libero e ispirato, la resa delle ombre attraverso il tratteggio, la prospettiva ottenuta non solo dalla rotondità dei corpi ma anche dalla giusta collocazione degli oggetti nello spazio, quei parametri per la valutazione dei dipinti, fissati alla fine del Seicento, nel quadro della Querelle des Anciens et des Modernes 43. Appunto alla Nozze Aldobrandini Caylus fa richiamo, nel I tomo del Recueil, a proposito del frammento di pittura ercolanese esaminato : " cet ouvrage m'a rappelé tout ce que je connaissais des peintures romaines trouvées à Rome cependant il est inferieur pour le dessins à la Noce Aldobrandini, qui par cette parti autant que pour la composition, tiendra longtemps le premier rang dans ce qui nous est resté de peintures antiques 44 ». Caylus, dunque, pur non avendole mai viste, avverte l'inferiorità delle pitture ercolanesi rispetto a quelle della capitale e la spiega con il fatto che difficilmente pittori di primo rango operanti in Roma si sarebbero recati in una città di provincia come Ercolano per dipingere pareti domestiche.

Nel tomo III del Recueil scrive : "Il m'est impossible de porter aucun jugement sur les peintures que la découverte d'Herculanum a mises au jour, je ne les ai point vues, et l'on ne peut décider sur la gravure : j'ignore même le degrés de confiance que mérite l'artiste chargé de cette entreprise ; mais en suivant la vérité des faits, il faut croire premièrement, que la peinture étoit déchue de son ancienne splendeur, lorsque ces fresques on été exécutées ; secondement, que les peintres qui ont travaillé dans cette ville, qu'on peut mettre que dans le second rang, en lui faisant même beaucoup d'hon-

42 CaYlus, Recueil, II, 1756, p. 109.

43 Le Nozze Aldobrandini facevano parte dei disegni tratti da pitture antiche, opera di Pietro Santi Bartoli, rinvenuti da Caylus a Parigi e da lui pubblicati, con la collaborazione di Mariette, nel 1757 nel Recueil des peintures antiques imitées fidèlement pour les couleurs et pour le trait, d'après les dessins coloriés faits par Pietro Sante Bartoli, contenente una serie di incisioni acquerellate, tratte dai disegni scoperti.

CAYlus, Recueil, I, p. 150. 
neur ; ces peintres, dis-je, n'étoient pas les plus habiles de la Grèce 45 ». Ma già nella memoria De la perspective, egli, a parte il dubbio espresso sul se una città di provincia come Ercolano potesse offrire un così gran numero di pitture ${ }^{46}$, e così belle come se ne diceva, aveva espresso l'avviso che non potessero essere state eseguite se non da artisti italici o da greci di secondo ordine, di parecchi secoli successivi ad Apelle. Ciò spiega anche il miglior livello, rispetto alla pitture, delle sculture rinvenute ad Ercolano, che ben potevano essere state eseguite altrove, sul quale in particolare si sofferma Cochin. Nella Lettre sur les peintures d'Herculanum, dopo aver, anche lui, rilevato che Ercolano era una piccola città di provincia, per cui difficilmente grandi pittori, di cui era necessaria la presenza per l'esecuzione degli affreschi, vi si sarebbero recati, aggiunge che la scultura e l'architettura vi avevano maggiore considerazione, perché legate al culto degli dei e all'idea dell'immortalità che esse promettevano agli uomini, mentre la pittura, confinata all'interno delle case per decorarne e abbellirne le pareti, esigeva un'attenzione meno scrupolosa ; Cochin conclude, nelle Observations, che, mentre in scultura era sufficiente rifarsi ad opere che già avevano raggiunto la perfezione, in pittura non vi erano che cattivi esempi da imitare, essendo scomparsi i resti della grande arte del passato. Caylus, a differenza di Cochin, non esamina l'aspetto della disomogeneità tra pittura e scultura ercolanese ; forse perché, in linea di principio, sostenitore della parità delle due arti, avendo esse per comune denominatore il disegno, e dell'omogeneità dei risultati da esse raggiunti nel medesimo tempo e nello stesso paese ${ }^{47}$. L'esistenza di validi modelli produce, ad avviso di Cochin, il miglior livello, come per la scultura, anche per l'architettura ercolanese rispetto alla pittura.

\section{2. Difetti e pregi delle pitture ercolanesi}

Al giudizio entusiasta degli italiani, tra cui Venuti, si contrappone quello, maggiormente meditato e teso ad evidenziare i difetti più che i pregi di quelle pitture, dei fran-

45 CaYlus, Recueil, III, p. 110.

46 Il numero delle pitture, secondo la ricostruzione fatta da C. Grell, Herculanum et Pompei dans les récits des voyageurs français du XVIII siècle, Napoli, 1982, sulla base dei dati desumibili dai resoconti dei visitatori, era rapidamente passato dai trentuno pezzi del 1739 (de Brosses), ai quattrocento del 1748 (d'Arthenay), ai seicento nel 1750 (d'Orbessan), agli ottocento nel 1756 (Barthélemy) sino a superare i mille nel 1762 (Winckelmann).

Caylus, nella memoria sulla scultura, osserva : «Il est cependant certain que l'unité des principes, le dessin qui est la base comune, enfin tout les rapports de la Peinture avec la Sculpture, étoient si parfaitement connus des Grecs, que, plusieurs de leur grands artistes ont pratiquement les deux arts ", in CAYLUS, De la Sculpture et des Sculpteurs anciens, selon Pline, letta in Accademia il 1 giugno 1753, in Histoire de l'Académie Royale des Inscriptions et Belles Lettres, Depuis son Établissement jusqu'à présent, Avec les Mémoires de littérature tirez des regîtres de cette Académie, XXV, Paris, 1717-1798, p. 302-303. 
cesi che, nel corso del Grand Tour, visitarono il museo di Portici. Cochin, nella Lettre, scrive in proposito : "vous connoissez les superlatifs des italiens, et vous avez assez demeuré dans leur Païs pour avoir éprouvé leur exagération ... 48 ».

Le valutazioni di Caylus in genere convergono con i giudizi espressi da Cochin e Barthélemy. Nel I tomo del Recueil, a proposito del frammento di pittura, parte di un più lungo fregio, riportato alla tavola LV (I), Caylus rileva : "On remarque dans ce qui en reste plus de liberté d'outils et de pratique que de science et de correction ; les détails, et surtout les extrémité, y sont fort négligés 49 ». Analogo il giudizio di Cochin nelle Observations : "ils sont presque tous très peu finis et peints à peu près comme nos décorations de théatre ; la manière en est assez grande, et la touche facile ; mais elle indique plus de hardiesse que de sçavoir 50 ». Nella lettera XI a Paciaudi del 5 marzo 1759 51, Caylus esprime il giudizio che le piccole donne all'aperto e i centauri (figure provenienti da Civita, l'antica Pompeja, c.d. villa di Cicerone, giudicate da Winckelmann " fluide come il pensiero e belle come di mano delle Grazie » e riprodotte nel I tomo delle Antichità Ercolanensi alle tavole 17-28) sono molto gradevoli ; apprezzamento, quanto alle "petites figures ", comune a Cochin, che, nelle Observations, scrive : "la touche plus hardi en est soutenue par un coloris plus vif que le reste du tableau : plusieurs, et ce sont les meilleurs, ont pour sujet une femme saisie par un Satyre 52 ", e lo estende ai " tableaux d'animaux et de fantaisie », e già nella Lettre aveva osservato : "ils sont touchés avec esprit ; le dessin en est très correct, et la couleur très bonne ... 53 ". Nella medesima lettera Caylus critica le pretese architetture « qui ne sont que des arabesques des plus mauvais et qu'on ne peut regarder que comme des opérations chinoises 54 ".

Già de Brosses aveva parlato di pareti " ornés d'architecture, mosaïque, carreaux de marbres ou peintures à fresque, en fleurs, ornemens légèrs, oiseaux ou animaux, d'une manière qui tient beaucoup de l'arabesque, mais plus légère 55 ". Critico in proposito è il giudizio di Cochin, che nelle Observations scrive : "Les moulures des corniches, des chapiteaux et des bases, très mal profilées, tiennent du goût des mauvaises Gothique ; la plupart des arabesque mêlées d'architecture sont aussi ridicule 273.

CaYlus, Recueil, I, p. 149.

C.-N. Cochin, J.-C. Bellicard, Observations sur les antiquités d'Herculanum, Paris, 1754, p. 42-43. 
que les desseins chinois 56 "; e prima ancora nella Lettre : " ... ils ont exprimé ces superbes bâtiments de mauvais goût, d'une façon extravagante, alongée, et déjà gotique, pour leur accorder au moins l'esprit prophétique 57 \%.

Le Antichità Ercolanensi definiranno queste architetture come capricciose ${ }^{58}$. La bizzarria, presente in alcune di quelle pitture, era peraltro già stata segnalata da de Brosses, che al proposito aveva osservato : « on remarque des choses si semblables à nos modes actuelles les plus bizarres, qu'on est prêt à les soupçonner d'y avoir été ajoutés après coup 59 ", cogliendo la singolare attualità di quei dipinti, i cui temi, attraverso quelli consimili delle grottesche, scoperte due secoli prima nella Domus Aurea, e diffusi dagli emblematisti, olandesi in particolare, erano pervenuti alla pittura dei moderni ${ }^{60}$.

Quanto al richiamo agli arabesques, il giudizio implicitamente negativo va riferito all'insistito decorativismo, di carattere orientalizzante, proprio dell'arte araba ${ }^{61}$. Il termine " gotico " va rapportato al connotato negativo che esso era andato assumendo, sin dai tempi del Rinascimento, come espressione di alcunché di barbaro contrario alla nobile semplicità dell'arte classica, del quale, secondo Mercier, proprio Caylus avrebbe propiziato l'abbandono : « le comte de Caylus - egli scrive - a resuscité parmi nous le goût grec et nous avons enfin renoncé à nos formes gothiques 62 ". La presenza di eccessi decorativi, anche nell'arte cinese, spiega poi l'accostamento tra arabeschi e cineserie operato da Caylus. Altri, poi, rileveranno la presenza nei dipinti ercolanesi anche di motivi decorativi egizi 63 , tanto da indurre taluno ${ }^{64}$ ad ipotizzare la presenza in

C.-N. Cochin, J.-C. BelliCARD, Observations, p. 148.

C.-N. Cochin, Lettre sur les peintures d'Herculanum, in J.-J. BARTHÉLEMY, Voyage, p. 280. Le pitture antiche di Ercolano e contorni incise con qualche spiegazione, II, 1760, p. 261, tav. XLVIII ; cf. sui caratteri "capricciosi » delle architetture presenti nelle pitture di Ercolano, F. BOLOGNA, "La riscoperta di Ercolano e la cultura artistica del settecento europeo", in F. Zevi (a cura di), Le Antichità di Ercolano, Napoli, 1988, p. 81-105.

De BROSSES, Lettre, p. 470-71.

Cf. il cap. Le antichità di Ercolano in M. Praz, Gusto neoclassico, Firenze, 1940.

Il termine " arabesco » è formata dalla parola " arabo » e nel nostro caso si riferisce ad ogni sorta di forma d'edificio fantastico, parti decomposte di architetture, utensili, mobili e oggetti d'arte. Quando gli arabi si espansero in nuovi paesi e vi costruirono grandi edifici, in essi il gusto e la conoscenza dell'antichità greca e romana erano scomparsi ; quando infine l'architettura (gotica) risvegliò l'amore dei monumenti e delle arti, il gusto dell'ornamento e della decorazione, non furono trovati altri modelli che non fossero quelli arabi. Cf. QuATREMÈRE DE QUINCY, voce "arabesque", in Dictionnaire Historique d'Architecture, I, Paris, 1832. Più in generale, si riferisce a motivi ornamentali semi fantastici.

L.-S. Mercier, Tableaux de Paris, Hambourg, 1781, cap. LXXXVIII.

A proposito dei motivi egittizzanti, cf. M. e A. DE Vos, "L'egittomania in pitture e mosaici romano campani della prima età imperiale", in Études préliminaires aux Réligions orientales dans l'Empire Romain, LXXXIV, Leyde, 1980, p. 26-28.

J.-C. R. DE SAINT-Non, Voyage pittoresque ou Description des royaumes de Naples et de Sicile, Paris, 1781-1786, II, p. 21. 
Ercolano, come prima a Roma, di artisti egiziani o addirittura a supporre che la Cina fosse stata colonia egiziana 65 .

Diverge invece il giudizio di Caylus da quello di Cochin, quanto alla presenza della prospettiva nella pittura ercolanese. Della prospettiva Caylus si era già interessato nella memoria De la perspective des Anciens, intesa a contrastare l'opinione di quanti, come Perrault, avevano negato che i greci conoscessero la prospettiva, ma ricca anche di più generali considerazioni sulla pittura.

Caylus ritiene che la prospettiva - consistente nel cambiamento che l'aria, per il colore, e la distanza, per il tratto, apportano sugli oggetti esposti alla nostra vista - fosse già nota ai greci e che la presenza di elementi prospettici nella pittura ellenistica, età di decadenza secondo il giudizio pliniano ${ }^{66}$, ne lascia presumere una pari presenza già nel periodo aureo dell'età classica. La maestria raggiunta dagli antichi, a suo giudizio, consentiva certamente loro di rendere le esigenze prospettiche, dal momento che anche la semplice matita, con l'utilizzo dei chiaroscuri, basta a rendere la rotondità dei corpi. Per ciò che concerne la pittura ercolanese, Caylus fa riferimento a un soggetto compositivo che, secondo voci correnti, rappresenterebbe " une scène parée de ses chœurs 67 ", per rilevare che la prospettiva, almeno quella del tratto, dovrebbe emergerne, supposto che quella del colore ne abbia sofferto. Affermazione che perfettamente si concilia con l'ascendente ellenistico della pittura ercolanese, pur se eseguita da pittori greci di secondo rango, e con la riconosciuta presenza della prospettiva nella pittura ellenistica 68 .

Cochin, nella Lettre, nega invece che i pittori di Ercolano ne fossero istruiti, come provano, a suo dire, i dipinti raffiguranti elementi architettonici e scrive che "n'etoient pas de grands peintres [...] ne connoissent que l'effet naturel de la vision [...] n'etoient

65 Tra questi, cf. G. SEIGNeuX DE CORREVON, Lettres sur la découverte de l'ancienne ville d'Herculane et de ses principales antiquités, Yverdon, 1770. Tesi sostenuta anche da de Guignes nella Mémoire dans laquelle on prouve que les Chinois sont une colonie egyptienne. Caylus, nella memoria Comparaison de quelques anciens monuments de diverses parties de l'Asie, si mostra invece più cauto ipotizzando un passaggio culturale dovuto all'imitazione di paesi intermediari. Per evitare poi che si ipotizzi un'origine cinese o indiana dei monumenti egizi, Caylus riconosce preventivamente in questi il carattere di assoluta originalità, evidente nelle piramidi egiziane di marcata semplicità, rispetto a quelle indiane così ricche di ornamenti. Entrambe le memorie sono pubblicate nell' Histoire de l'Académie Royale des Inscriptions et Belles Lettres. Plinio, Naturalis Historia XXXIV, 51-52.

67 CaYlus, De la perspective des Anciens, in Histoire de l'Académie Royale des Inscriptions et Belles Lettres, XXIII, p. 323.

La tesi che l'arte greca conoscesse già la prospettiva è ora largamente condivisa. Bianchi Bandinelli osserva in proposito che l'arte greca « è l'unica, infatti, che abbandona la ripetizione degli schemi figurativi fissi e simbolici ; l'unica che inventa lo scorcio e la prospettiva e il colore locale per afferrare l'aspetto realistico delle cose. Essa si pone precocemente sulla via del naturalismo per realizzarlo pienamente nell'età ellenistica " in R. BIANCHI BANDINELLI, Introduzione all'archeologia, Bari, 1976, p. 18. 
point assez instruits des règles de la perspective 69 ", in particolare osservando che « les tableaux d'architecture [...] n'ont aucune perspective 70 \%. Ma sfuma la sua posizione nelle Observations, dove rileva che "l'architecture s'y trouve en quelque façon mise en perspective, mais d'une manière qui prouve que les auteurs de cette composition n'en sçavoient pas la règle ${ }^{71}$ ».

\section{3. Scelta dei colori e tecniche pittoriche}

Quello della scelta dei colori e delle tecniche pittoriche è forse l'aspetto più originale della ricerca caylusiana.

Sulla prima interessante è quanto Caylus scrive nel I tomo del Recueil a proposito del frammento di pittura, parte di un più lungo fregio, riportato alla tav. LV (I) ${ }^{72}$. Tale frammento, in cui il colore del fondo è quasi nero, induce il conte a ritenere che gli antichi non amassero i colori chiari, soprattutto nella decorazione delle loro case. Affermazione che invero sembra contrastare con quanto da lui stesso osservato nel $D e$ la perspective des Anciens, a proposito dei colori chiari, che gli antichi, a differenza dei moderni che spesso utilizzano quelli scuri, privilegiavano per poter riportare nel dipinto, con eguale risalto, tutti gli oggetti presenti nella scena da ritrarre. Ma l'apparente antinomia si risolve considerando quanto Caylus precisa nella memoria : "ce qui nous reste, je parle toujours avant de la découverte d'Herculanum, représentent en général les figures seules, executées sur des fonds vagues et d'un seul couleur 73 ", accennando all'inversione di tendenza proprio ad opera della pittura ercolanese. Caylus, nel citato passo del Recueil, riferisce ancora di aver esaminato un gran numero di piccoli frammenti di muri ercolanesi, il cui intonaco era dipinto con arabeschi e ornamenti piuttosto grossolani, e con colori decisi, come il rosso scuro contrastato dal nero. La predilezione per le tinte più scure, dovuta in primo luogo alla maggiore durata e resistenza agli agenti atmosferici, sarebbe finalizzata anche ad evitare l'eccessivo riverbero della luce solare, determinato dall'uso del bianco e dei suoi derivati, al qual proposito Caylus arriva ad ipotizzare che la vista degli antichi, soprattutto gli abitanti di Ercolano, fosse particolarmente delicata. Le osservazioni di Caylus si ricollegano alla tesi, a lui cara, dell'influenza del clima sulle arti. Nel Recueil I egli sostiene che sul goût d'une nation incidono le condizioni ambientali, tra cui principalmente il clima; tesi, questa, già enunciata da Montesquieu (Esprit des lois) e poi ripresa da Winckelmann

69 C.-N. COCHIN, Lettre sur les peintures d'Herculanum, in J.-J. BARTHÉLEMY, Voyage, p. 280.

70 C.-N. Cochin, Lettre sur les peintures d'Herculanum, in J.-J. BARTHÉLEMY, Voyage, p. 279.

73 CaYlus, De la perspective des Anciens, in Histoire de l'Académie Royale des Inscriptions et Belles Lettres, XXIII, p. 327. 
(Gedanken), il quale ritenne sia il clima sia il regime politico fattori determinanti l'eccellenza dell'arte greca. L'influenza del clima sulle arti è ripetutamente richiamata da Caylus a proposito degli etruschi, avvantaggiati da quella mitezza del clima, che nei secoli avrebbe favorito in Italia lo sviluppo delle arti ${ }^{74}$. All'influenza sul modo di edificare che ogni paese subisce ad opera della natura e del clima si riferisce quanto Caylus scrive nella memoria sull'architecture ancienne a proposito dei muri molto spessi costruiti dagli egizi per proteggersi dall'intensità del sole e dalla natura dei materiali da costruzione, come ad esempio la durezza delle pietre, i cui grani sono più compatti nei paesi caldi, la sabbia più secca, oltre l'aria più eguale e esente da umidità, fattori tutti che contribuiscono al tipo di costruzioni e alla durata degli edifici di alcuni paesi. Caylus ritorna ancora sull'uso del fondo nero nella tav. LVI (I) ${ }^{75}$, a proposito del frammento ercolanese portatogli da Soufflot, per evidenziare come il colore risulti più brillante per effetto del nero dello sfondo.

Alle varie tecniche pittoriche adottate dagli antichi Caylus dedica particolare attenzione, certamente alimentata dalle scoperte ercolanesi, con un'ampia estensione di campo, dall'encausto, alla pittura su legno a tempera, all'affresco per la pittura murale.

Nel De la perspective des Anciens egli indugia in particolare sull'affresco per evidenziare la difficoltà della preparazione dell'intonaco e dei colori nella misura adeguata a dipingere l'estensione dell'intonaco predisposta, e della visione d'insieme da tenere necessariamente presente nelle pitture murali di vasta estensione eseguite per superfici successive.

Nel 1752 in una comunicazione all'Académie des Inscriptions et des Belle Lettres intitolata Réfléxions sur quelques chapitres du XXXVe livre de Pline, letta il 21 novembre ${ }^{76}$, Caylus suggerì la possibilità di ricostruire il procedimento della pittura ad encausto, come descritta da Plinio. Seguendo la descrizione pliniana e con l'aiuto del chimico Majault, egli era riuscito ad ottenere con la mescolanza di cere e pigmenti un preparato sufficientemente liquido tale da conservare la fluidità per il tempo adeguato all'esecuzione del dipinto e da poter essere facilmente steso con il pennello, perché poi, terminato il dipinto e posto lo stesso dinanzi al fuoco, la cera sottostante lo strato colorato penetrasse i pigmenti e risalisse alla superficie. Ed un quadretto raffigurante una testa di Minerva, eseguito secondo tali indicazioni da Joseph-Marie Vien, fu presentato nel 1754 all'Accademia e accolto con grande favore. Uno scritto anonimo, l'Histoire et le secret de la peinture en cire, presto attribuito a Diderot contestò non il metodo ma la paternità di esso, sostenendo che era già stato usato dal pittore Jean-Jacques Bachelet nel 1749 ; Caylus si difese con la Mémoire sur la peinture à l'encaustique et sur la peinture à la cire, par M. le comte de Caylus et M. Majault, letta nel novembre del 175477. 
Di notevole interesse è poi quanto Caylus osserva nel tomo IV del Recueil in relazione all'uso del minio rosso nelle pitture ercolanesi. Il fondo di molte pitture sarebbe ottenuto, secondo il conte, dall'uso del minium disteso sulla parete, ricoperto da uno strato di cera, riscaldato poi da una sorgente di calore, secondo un procedimento descritto da Vitruvio (libro VII) che il conte preferisce a quello simile indicato da Plinio (Nat.Hist. XXXIII). Le figure invece erano dipinte successivamente e non facevano corpo con il colore del fondo, di qui l'ottima tenuta di questo, attraverso il procedimento indicato, e invece quella mediocre delle figure, soggette a scrostarsi. A giudizio di Caylus « l'explication des Peintures d'Herculanum, nous apprend que les Ouvrages retrouvés dans les fouilles de Portici, sont presque tous exécutés de cette manière ; ainsi nous ne pouvons douter que les Anciens n'ayent peint à la détrempe sur les murailles ; c'est-à-dire, qu'ils n'ont pas toujours employé la Fresque 78 ».

In realtà, nel Settecento opinione diffusa era che le pittura ercolanese, escluso l'encausto, fosse in prevalenza a tempera, eseguita ciò̀ con colori non facenti corpo con l'intonaco, piuttosto che a fresco. E alla natura dei dipinti era legato il problema dell'impiego delle vernici conservative, in particolare quella proposta da Stefano Moriconi e suggerita da Marcello Venuti, che diede esiti non soddisfacenti, perché nel 1766 alcune delle pitture così consolidate iniziarono a scrostarsi. In generale Caylus, come ricorda Zevi ${ }^{79}$, si era già manifestato contrario all'uso delle vernici, ciò in linea con il suo rigorismo, avverso a qualunque rimedio potesse, comunque, alterare il reperto antico, inficiandone i valori di testimonianza e significazione.

\section{4. Un giudizio equilibrato : tra arte e antiquaria}

Caylus, sulla base delle notizie acquisite e dei pochi frammenti pervenutigli, pur non avendole potuto direttamente vedere, riesce tuttavia a formulare un approfondito giudizio in merito alle pitture ercolanesi, tra arte e antiquaria, i due versanti della sua ricerca, che gli consentirono, in anticipo su Winckelmann, di superare gli angusti confini dell'erudizione antiquaria del suo tempo. Merito che a giusta ragione gli riconosce Paciaudi nella lettera LVII del 27 aprile 1761 : « vous réunissez deux qualités qui vous mettent au dessus de tous les autres antiquaires, à la connaissance de l'antiquité vous joignez celle des arts 80 ".

78 CAYLus, Recueil, IV, p. 223-226 ; cf. sull'uso dell'encausto anche nelle pitture parietali A. ReInaCH, Recueil Milliet, Textes grecs et latins relatifs à l'histoire de la peinture ancienne, Paris, 1921 ; sull'impiego misto delle due tecniche nelle pitture pompeiane, v. S. FERRI, Plinio il vecchio. Storia delle arti antiche. Naturalis Historia (libri XXXIV-XXXVI), Milano, 2001. Ercolano, Napoli, 1988, p. 11-38. 
Caylus si giova della sua approfondita conoscenza della pittura antica, alla quale aveva dedicato, oltre le già ricordate De la perspective des Anciens, Réfléxions sur quelques chapitres du XXXVe livre de Pline [prima parte], Réfléxions sur quelques chapitres $d u$ $X X X V^{e}$ livre de Pline [seconda parte] e Du caractère et de la manière des Peintres Grecs [terza parte], la memoria Description de deux tableaux de Polygnote, donnée par Pausanias e quella Sur le tableau de Cébes, sur l'autre de Coryce et sur la tableaux de Philostrate. Né va dimenticata la sua conoscenza dell'arte pittorica, privilegiata rispetto alla scultura, alla quale aveva dedicato alcuni fondamentali studi (la dissertazione Sur les dessins des maîtres; le comunicazioni Réflexions sur la peinture, Sur ce qu'on appelle la manière et le moyens de l'éviter; Sur l'armonie et sur la couleur), che contengono le sue posizioni in relazione ai grandi temi del dibattito estetico settecentesco.

Caylus, molto realisticamente, si rende conto della difficoltà di emettere giudizi sulla pittura antica, dato lo stato dei dipinti pervenuti. Nel De la perspective des Anciens si chiede come si faccia ad attaccare o a difendere una pittura di cui si è a conoscenza solo attraverso i resti di dipinti murari ancora conservati, certamente inferiori alle grandi opere elogiate dagli autori antichi ; un'analoga cautela, proprio a proposito delle pitture ercolanesi, aveva suggerito de Brosses, rilevando, quanto al difetto del colore di quelle pitture, "il semble que ce que l'ont peut le plus justement reprocher aux anciens est le défaut d'ordonnance et de distribution des masses : quand le coloris d'une pièce est entièrement perdu, est-il bien aisé de juger de sa perspective, de son clair obscure et des couleurs locales 81 ?"

Caylus, nel generale clima di attesa per le scoperte di Ercolano - « il faut espérer - egli scrive - qu'après une si longue attente nous serons instruits de toutes ces découvertes 82 " - si persuade tuttavia che i dipinti ercolanesi, opera di pittori greci di secondo rango, non sono all'altezza delle grandi opere del passato, ma inferiori anche a quelli romani, Nozze Aldobrandini in particolare, e non sono in grado di fornire, nel quadro della non sopita Querelle des Anciens et des Modernes, validi modelli ai pittori moderni. Tant'è che la pittura ercolanese in realtà esercitò un'influenza limitata sui moderni, pur essendosi ad essa ispirati il Vien del La Marchande d'Amours e il fin troppo celebrato Mengs, cui Mariette 83 rimproverò l'eccessiva freddezza dello stile, non diversamente da quanto fecero Cochin e Barthélemy nei confronti della pittura ercolanese. Caylus avverte che questa pittura, pur se di ascendenza ellenica, è lontana dal vagheggiato ideale della nobiltà e semplicità della pittura greca, cui quella dei moderni dovrebbe ritornare per l'auspicato superamento del degrado prodotto dallo stile rocaille. 1860. 
Il giudizio del conte si allinea sostanzialmente a quello di Cochin e Barthélemy, non dovendosi però dimenticare che alcune sue acute osservazioni sono formulate nel De la perspective, anteriore nel tempo agli scritti dei suoi due maggiori referenti; se ne discosta poi per qualche aspetto, come quello della prospettiva, e si arricchisce di originali osservazioni sulla scelta dei colori e sulle tecniche pittoriche. In definitiva il giudizio di Caylus sulle pitture ercolanesi, pur se condizionato dalle limitate conoscenze che poté averne, appare persuasivo : ne emerge il quadro di una pittura minore, di carattere prevalentemente decorativo, certamente non in grado, così come la pittura parietale in genere, di evocare i fasti della grande pittura su tabulae del passato ; disegno, colore, espressione, disposizione delle figure nello spazio lasciano a desiderare ; certamente da tali pitture non può trarsi alcun insegnamento per i moderni, come già Cochin aveva da par suo immediatamente intuito. Di ciò Caylus, senza lasciarsi trascinare da facili entusiasmi, come quelli di Venuti o dello stesso Winckelmann, quando gli "fa velo " la passione per alcuni soggetti, si rende conto. E tuttavia ne segue, con la consueta attenzione per gli aspetti tecnici, i colori e il loro impiego sulle pareti, cerca di individuare le ragioni delle scelte e ne valuta i risultati.

Egli considera importante, di per sé, ogni cosa, dipinti compresi, che Ercolano ha miracolosamente restituito, dove e come era al momento dell'eruzione vesuviana, eccezionale testimonianza della cultura materiale di quella città. Ed è in tale direzione che si muove la ricerca caylusiana, alla riscoperta di una nuova misura dell'antico, che è suo merito l'aver per primo indicata. Significativo in tal senso è quanto egli scrive in relazione al compasso pubblicato alla tavola LXXXV del V tomo del Recueil « Herculanum peut seul nous donner un cours des utensiles employés par les Romains, mais souvent on néglige dans les grands trésors les petites pièces de monnaies et, sans être conduit par un principe de charité, on laisse de quoi glaner aux pauvres 84 „. È la sua predilezione per i piccoli oggetti, rispetto alle grandi opere d'arte, giacché " plusieurs de ces guenilles ont fait trouver des opérations méchaniques très singuliers et très utiles aux artistes pour lesquels j'avoue que j'aime plus à travailler 85 ». Di qui l'attenzione che Caylus porta allo strumentario domestico, direzione nella quale si muoveranno le tavole dell'Encyclopédie e, anni dopo, le Tables des usages civils et militaires trouvé à Pompéia et à Herculanum di Francesco Piranesi.

La pittura parietale, alla pari degli altri oggetti rinvenuti nella città dissepolta, diventa una preziosa fonte di suggerimenti quanto ai motivi decorativi minori per " tutti gli orefici, bigiuttieri, pittori di carrozze, di sovrapporte, tappezzieri, ornamentisti 86 ", come scrive da Parigi a Tanucci l'abate Galiani, nella famosa lettera del 1767, nella quale riporta anche l'equiparazione, corrente in quella città, dell'espressione à la

Caylus, Recueil, V, 1762, p. 239.

CaYlus, Correspondance, I, p. 9.

La lettera di Galiani a Tanucci è riportata da F. BolognA, "La riscoperta di Ercolano", in F. Zevi (a cura di), Le Antichità di Ercolano, Napoli, 1988, p. 84. 
grecque con quella à Erculanum. Ed è proprio in tale settore - mobilia, suppellettili e sistemi decorativi dell'architettura neoclassica - che Ercolano eserciterà una decisiva influenza.

\section{Le pubblicazioni ufficiali : la critica degli « Ercolani »}

\section{Il Catalogo degli antichi monumenti dissotterrati nella discoperta città d'Ercolano di} Baiardi, pubblicato, totalmente privo di tavole, nel 1756, dopo il perentorio ordine del sovrano di interrompere la serie di cinque volumi del Prodromo, si segnalò per l'eccessiva verbosità delle descrizioni, l'omesso inserimento degli oggetti nei loro contesti. Ad esso Caylus dichiara di essersi rifatto, a proposito dei disegni della tavola XXXVII, pubblicata nel III tomo del Recueil, riconoscendo " que le catalogue du Cabinet de Portici m'a mis en état d'entrer dans le détails du plus grand nombre de ces morceaux et de rapporter plusieurs eclaircissement, que les dessins ne peuvent donner. J'ai donc profité des notes du Catalogue, et pour les gravures de ces trois planches, je renvois à la description qu j'ai trouvé dans cet Ouvrage 87 ». Caylus, dunque, non muove critiche al catalogo di Baiardi. Decisamente critico è invece riguardo alle Pitture antiche di Ercolano e contorni, incise con qualche spiegazione, opera affidata all'Accademia Ercolanense, fondata nel 1755, dopo l'insuccesso della pubblicazione dei primi cinque volumi del Prodromo baiardiano. Il primo volume dell'opera, destinata per volontà del sovrano a pochi privilegiati, d'alto rango, e negata anche a studiosi di riconosciuta fama, fu pubblicato nel 1757 , con una difesa tra l'altro dalle critiche cui erano state sottoposte le pitture ercolanesi, delle quali, pur riconoscendosene alcune cadute di stile, veniva confermato il complessivo valore 88 .

A proposito del I volume, Caylus, nella lettera XI a Paciaudi del 5 marzo 1759 89, al teatino che gli aveva chiesto cosa ne pensasse (" Avez-vous encore vu les peintures d'Herculanum ? Vous ne m'en avez jamais rien dit, et je ne sais pas ce que vous en pensez. Éclairez-moi sur ce point, vous qui voyez si bien 90 ") esprime il giudizio che il volume sia stato eseguito in modo assai ineguale per ciò che concerne le incisioni e che sia poco affidabile; critica poi l'inserzione, in opera di tale importanza e soprattutto nel I volume, delle pretese architetture, da considerarsi nulla più che brutti arabeschi ; trova le spiegazioni farcite di notizie inutili e ripetute; esprime l'avviso che nessuno di coloro che hanno lavorato nell'opera comprenda le arti e dica cose che ad esse si addicano.

87 CAYLUS, Recueil, III, p. 143.

88 Cf. sui vari aspetti della pubblicazione delle antichità di Ercolano, il cap. Ercolano, in C. LENZA, "La cultura architettonica e le antichità".

90 P. M. PACIAUDI, Lettres, p. 26. 
Quanto al secondo volume, pubblicato nel 1760, nella lettera a Paciaudi XLIX del 16 febbraio 1761, Caylus scrive "ce n'était pas la peine de nous tenir si longtemps le bec dans l'eau pour le IIe tome d'Herculanum, puisque il est si mal exécuté 91 ", riferendosi al giudizio fortemente negativo del teatino (" J'ai vu le deuxième volume des peintures d'Herculanum : il fait pitié. Il est dessiné diaboliquement, gravé sans goût, sans intelligence, et sans aucune grâce. C'est un malheur que des choses si rares soient entre les mains de gens si ignorans, et qu'un roi soit si mal servi 92 »). Caylus nella lettera LII a Paciaudi del 23 marzo 176193 scrive che il secondo volume gli sarà fatto vedere da Galiani non appena gli sarà pervenuta la cassa con gli « Ercolani », nome con cui in Francia venivano denominate le antichità ercolanensi ; cassa che pervenne all'abate nell'aprile del $1761{ }^{94}$; quello di Caylus è dunque un giudizio "de relato " pur non essendo da escludere che il conte abbia potuto già prima visionarne un esemplare. Paciaudi, nella lettera LVII del 29 aprile 1761, ritorna sul secondo volume delle antichità, criticandone le incisioni, nelle quali " tout y est trop maniéré » e, in generale, l'accademia di Napoli, che « ... est composée d'homme savans; mais il y a tout au plus deux bons antiquaires. Comment voulez-vous que leurs observations puissent contenter un homme consommé, comme vous l'êtes, dans cette partie ? $95 \%$.

In relazione al III volume delle Antichità, pubblicato nel 1762, Caylus ricorda, nella lettera a Paciaudi LXXXIV del 4 marzo 1764, di averlo ricevuto dal principe Ferdinando di Parma ${ }^{96}$ e nella successiva lettera LXXXIX del 1 aprile 1764 rimarca la differenza tra il favore dimostrato dal giovane principe e la " finezza " (in italiano nel testo) con cui il dono gli è stato fatto e "l'abbé Galiani, qui vient de temps en temps m'épier au sujet d' Herculanum 97 ». Non si rinvengono precisi apprezzamenti su questo volume.

Comunque il giudizio sulle Antichità è, complessivamente, negativo e ne emerge il disegno di Caylus di sminuirne l'importanza, sia per la materia in parte concorrente con il suo Recueil, nel quale i reperti ercolanesi sono inclusi nella classe greca, sia per il noto risentimento che egli nutre nei confronti di governanti e eruditi napoletani. Proposito che traspare evidente dalla pubblicazione nel 1754 della traduzione in francese della lettera che Winckelmann, due anni prima, aveva indirizzato al conte di Brühl. La lettera, contenente una severa critica agli errori commessi nelle pubblicazioni erudite sugli scavi ercolanesi e in particolare dal Martorelli nel De theca calamaria quanto ai papiri e alla scrittura antica, suscitò la reazione degli accademici napoletani e dello

Caylus, Correspondance, I, p. 243.

P. M. PACIAUDI, Lettres, p. 218.

CaYLus, Correspondance, I, p. 254-261.

CaYlus, Correspondance, I, p. 257, n. 2.

P. M. PACIAUdI, Lettres, p. 235.

CaYlus, Correspondance, I, p. 426-433.

CaYlus, Correspondance, I, p. 458. 
stesso Tanucci 98 ; Caylus nella lettera a Paciaudi CXXXIII, senza data (ma tra il 10 e il 21 maggio 1765), scrive a tal proposito : " le mécontentement de Martorelli et des académiciens ne peut nous conduire qu’à de plus grandes vérités, et la colère fait souvent dire ce que le sang-froid est bien éloigné d'avouer 99 ". La pubblicazione della lettera di Winckelmann, in riferimento alla quale Caylus si era già espresso favorevolmente ("Je suis content de lui par rapport à Herculanum 100 »), raggiunse l'evidente scopo di screditare le Antichità ercolanensi, ma anche quello, ambiguo e meno trasparente, di incrinare i rapporti tra lo studioso tedesco e gli eruditi napoletani ${ }^{101}$.

L'anno seguente sarà pubblicato il IV volume delle Antichità ; Caylus, nella lettera a Paciaudi CXLVI del 19 agosto 1765, si dichiara contento della relativa prefazione, in quanto in essa gli viene riconosciuto qualche merito ${ }^{102}$. Il riferimento è alle due tessere in osso per l'ingresso a teatro, trovate ad Ercolano, quella con la sola maschera comica, che il conte ha ritenuto attinente alla rappresentazione di una commedia, e altra con il nome di Eschilo, che viene riferita alla rappresentazione di un'opera del tragico greco 103 .

Il tono di questa lettera appare più distaccato e meno polemico nei confronti degli autori delle Antichità, il risentimento, se non placato, più tenue ; Caylus, già malato gravemente all'inizio del 1765, morrà a settembre dello stesso anno.

Winckelmann, l'altro severo censore del modo con cui la vicenda Ercolano era stata gestita, ritenuto dallo stesso Tanucci meritevole di perdono in considerazione della sua lunga permanenza romana, col tempo rientrerà nelle grazie sovrane e, in occasione della sua ultima visita a Napoli del 1767, Ferdinando IV gli farà dono di una copia del tomo V delle Antichità, contenente 120 bronzi di Portici, e Winckelmann assumerà l'incarico di revisionarlo.

L'anno successivo si concluderà la vita dell'abate prussiano. I suoi meriti, già lui vivo, riscuoteranno i più alti riconoscimenti ; Caylus, condannato ad un lungo oblio dopo la sua morte, dovrà attendere due secoli per vedere riconosciuti i propri.

\begin{tabular}{l} 
Enrica PAGANO \\
\hline Viale Antonio Gramsci 14, \\
80122 Naples (Italie) \\
enrica.pagano@libero.it
\end{tabular}

98 Cf. sulle polemiche suscitate a Napoli dalla pubblicazione della lettera, F. STRAzzULLO, Nota Introduttiva, J.J. WInCKELMANN, Le scoperte di Ercolano, Napoli, 1981.

99 CAYLus, Correspondance, II, p. 123-124.

100 CaYlus, Correspondance, I, p. 410.

101 Cf. E. DeCulTOT, Johann Joachim Winckelmann. Enquête sur la genèse de l'histoire de l'art, Paris, 2000.

102 CAYLus, Correspondance, II, p. 162-165.

103 Le pitture antiche di Ercolano e contorni incise con qualche spiegazione, IV, 1756, Prefazione, p. VII, n. 8. 